\title{
D emocracia y gobernabilidad de las grandes ciudades en Europa: una comparación internacional
}

Vincent H offmann-M artinot*

El final del siglo $x x$ ha venido a significar una ruptura en la evolución de los sistemas políticos de las grandes ciudades, tan fundamental como el período de cambio posterior a la segunda guerra mundial. En un escenario de crisis económica persistente, de cambios en los valores y en los comportamientos socio-políticos y de un creciente distanciamiento entre ciudadanos y sus re presentantes, las instituciones existentes parecen haber agotado su capacidad de adaptación a los retos de las sociedades. A través de una asombrosa convergencia, el deseo de una renovación profunda de la gobernabilidad urbana se expresa bajo formas diferentes en el conjunto de los países europeos: en Francia, mediante una presión más o menos organizada en favor de una superación de la «congregación de notables» resultante de las reformas de descentralización; por la generalización, más allá del Rin, del modelo considerado más democrático y más eficiente del Sur de Alemania; en Italia, a través de medidas destinadas a reforzar la legitimidad de la clase política local; en los países escandinavos, por una política de autonomización de la acción pública local que pasa por la experimentación de la fórmula denominada de los «municipios libres»; en Gran Bretaña, por estrategias que preconizan hacer saltar la tapadera de la centralización thatche riana; en España, por la ola de propuestas dirigidas a «reinventar» el gobierno local. Esta democracia urbana, cuya característica principal y superior consiste, en principio, en asegurar el mayor acercamiento entre los ciudadanos y sus gobernantes, se halla sometida a un aumento de las insatiffacciones y a una puesta en tela de juicio de sus fundamentos y modalidades de actuación.

\section{¿Por qué comparar las democracias urbanas a escala internacional?}

Un equipo internacional de investigadores que ha elaborado el estudio comparado Démocraties urbaines ${ }^{1}$ (G ABRIEL y otros, 2000) parte de la siguiente constatación: a partir de los años ochenta, especialmente, estamos asistiendo en numerosos países a una auténtica crisis de la democracia en las grandes ciudades cuyas manifestaciones son múltiples: crecimiento del abstencionismo electoral, declive de la legitimidad de los elegidos municipales, complejidad de las expectativas y de las demandas de los grupos sociales que son cada vez más heterogéneos, relativa desvitalización de los centros de las ciudades en beneficio de los municipios de la periferia, reforzamiento de la segregación social, tensiones financieras crecientes entre cargas en aumento y recursos en retroceso y retirada general de los actores estatales.

D e este modo, el espectro que parece atormentar a más de un alcalde de gran ciudad europea es el de las metrópolis americanas caracterizadas por un declive rápido, cuando no espectacular, del centro de la ciudad, donde coexisten islotes de riqueza (barrio de negocios) y barrios en decadencia y rodeada de un cinturón de áreas periféricas habitadas de manera relativamente homogénea por la clase media. La ampliación registrada en los últimos años de las desigualdades socioeconómicas y de los con- 
flictos de reparto inter e intramunicipales se encuentra en el centro de las inquietudes e incertidumbres expresadas, de manera asombrosamente convergente y simultánea, por los alcaldes de las grandes ciudades alemanas y francesas en sus manifiestos de 1994 (Kron AW ITTER, 1994; Association des M airies de Grandes Villes de France, 1994).

Ante esta acumulación de transformaciones fuertemente perturbadoras, tanto socioeconómicas como políticas, de la gobernabilidad urbana, el programa D émocraties urbaines tenía como objetivo comprobar el estado de losfundamentos, de las estructuras y de los cambios de la democracia de las ciudades de más de 100.000 habitantes en doce países industrializados que representan a Europa (Alemania, Francia, Gran Bretaña, Italia, Países Bajos, Dinamarca, Finlandia, N oruega y Suecia), América del N orte (Canadá y Estados Unidos) y Asia (Japón).

\section{Los efectos políticos de la transformación socioeconómica de las ciudades}

H asta ahora no se había llevado a cabo ningún proyecto internacional de este género. En verdad, sólo se disponía de trabajos comparados generales desde el punto de vista institucional que se referían no sólo a las ciudades, sino a las entidades locales en general y, en particular, a su relación con el Estado. Ahora bien, las grandes ciudades experimentan dinámicas sociopolíticas y cambios institucionales que las diferencian cada vez más de las otras autoridades locales. La aceleración de la metropolización es una de las causas principales que incita a los respon sables político-administrativos de las grandes aglomeraciones a desarrollar y reforzar la organización colectiva de sus intereses y sus estrategias de lobbying a nivel nacional o supranacional: basta con mencionar el peso, a menudo determinante en la elaboración de numerosas políticas públicas, de estructuras tales como las US Conference of $M$ ayors de los Estados Unidos o el Städtetag en Alemania, la creación de redes de ciudades mono o plurisectoriales, la promoción y la defensa por las asociaciones nacionales de grandes ciudades europeas de la especificidad de sus «problemas» ante las instancias comunitarias.

Las transformaciones sociales globales a las que se hallan sometidas las grandes ciudades de los países industrializados - ya sean alemanas, francesas, japonesas o americanas- con frecuencia escapan en gran medida a la posibilidad de control por parte de las autoridades municipales. Diversos factores de cambio social contribuyen a modificar en profundidad la naturaleza de los sistemas políticos urbanos, en especial:

- El paso de una sociedad industrial a una economía de servicios, así como la internacionalización de las formas de producción y de distribución de bienes y servicios.
- El incremento de la movilidad regional en el interior de las sociedades y entre ellas.

- La revolución tecnológica y, principalmente, el desarrollo de la difusión de nuevas tecnologías de comunicación, de producción y de distribución de recursos tecnológicos e intelectuales.

- La elevación del nivel de enseñanza.

- El cambio de los valores y de los estilos de vida.

Estas transformaciones sociales producen efectos en los diferentes niveles del proceso político urbano, es decir, en los outputs, en los inputsy en la conversión o en las interacciones entre inputsy outputs. En lo que se refiere a los outputs, los cambios socioeconómicos y culturales suponen más demandas de bienes y de servicios (revolution of social expectations), pero, al mismo tiempo, hacen surgir nuevos retos políticos, a menudo contradictorios, en la agenda tanto nacional como local (problemática de la «nueva política», (CLARK y H offm Ann -M ARTIN OT, 1998). La evolución de los tipos de demandas articuladas por los actores individuales y colectivos está vinculada a rei vindicaciones de innovación en el seno de las estructuras decisionales (agentes de conversión), tales como una mayor eficiencia de las asambleas locales, un estilo de decisión más transparente, una redistribución de las responsabilidades entre los electos y los administradores, nuevas formas de gestión y de prestación de servicios, una asignación más eficiente de los recursos a los outputs y una diversificación de la selección y de la socialización de los responsables administrativos (CAN ALES AlIENDE, 2000).

Si consideramos ahora la dimensión de los inputs, el denominador común de las diversas transformaciones de la relación entre gobernantes y gobernados es una transformación del comportamiento individual que conduce a nuevas configuraciones de mediación de los intereses a escala de las ciudades y de los sistemas políticos nacionales. Los responsables municipales se enfrentan a unos ciudadanos cada vez más autónomos, informados y activos que plantean reivindicaciones de participación directa en los procesos decisionales locales. Los desafíos a la democracia urbana que resultan de la evolución de las actitudes y de los comportamientos políticos con respecto al sistema político local se acentúan por el hecho de que las organizaciones y las estructuras tradicionales de mediación de los intereses no son consideradas por los ciudadanos como los únicos puntos de acceso pertinentes al sistema político. D ado que las elites políticas no han logrado todavía adaptarse completamente a esta transformación del medio político y satisfacer el conjunto de las demandas que se les dirigen, cada vez son más los ciudadanos que ponen en duda su capacidad para responder a sus expectativas y tienden a alejarse de sus representantes políticos.

Además de los recursos de los que disponen las autoridades municipales para enfrentarse a esta presión creciente de las de- 
mandas sociales, la calidad de la democracia en las grandes ciudades depende de la evolución de su base socioeconómica. En algunos países, el estrato social que más activamente participa en la política urbana tiende a abandonar el centro de las ciudades, que concentran cada vez más los segmentos más apáticos de la población. Esto tiene por consecuencia un debilitamiento del compromiso municipal y un desequilibrio de la relación entre los ciudadanos y sus representantes, por una parte, y las autoridades municipales, por otra. La existencia de una población políticamente activa constituye una característica esencial de una democracia estable y eficiente, no sólo en el nivel municipal, sino también en el nacional, y la posibilidad de una crisis de la democracia local tiene implicaciones que superan naturalmente los límites de una determinada localidad.

\section{Las preguntas de la comparación internacional}

$H$ ay que hacerse tres preguntas importantes cuando se considera el estado actual y el futuro de las democracias urbanas:

- ¿Por qué la transformación de las grandes ciudades mere ce una atención especial en el análisis del impacto del cambio social en los sistemas políticos en general?

- ¿Por qué interesarse especialmente en el estado y la evolución de la democracia en las grandes ciudades?

- ¿Por qué hay que tener una perspectiva comparada en este enfoque de la democracia urbana?

Las grandes ciudades y las áreas metropolitanas juegan un papel particularmente importante en los estudios del cambio social, no solamente porque la mayor parte de la población de las sociedades contemporáneas se concentra en las regiones urbanas, sino también porque allí emergen numerosos problemas sociales y se manifiestan con crudeza. El análisis del cambio social en las grandes ciudades permite, por consiguiente, aclarar y comprender de forma privilegiada los problemas futuros del conjunto de una determinada sociedad.

Los procesos globales de cambio social, económico y cultural observados en las sociedades industrializadas implican numerosas consecuencias políticas, a escala tanto nacional como local, en los diferentes estadios del ciclo de las políticas (inputs, conversión y outputs). La evolución de la democracia local y urbana constituye un reto central de los debates que se refieren a las implicaciones políticas de cambio social. Aunque algunos modelos de gobierno local (el modelo británico, en particular) conceden una importancia primordial a la eficiencia en la prestación de servicios, el papel del gobierno local no puede reducirse a esta función de producción de bienes y servicios si no es al precio de una pérdida sustancial considerable. En la mayoría de las democracias pluralistas, la democracia urbana está muy consolidada, sobre todo gracias a unos mecanismos de participación más 0 menos desarrollados.

La forma en que las grandes ciudades se gobiernen en un futuro próximo está en el núcleo de la evolución de las democracias contemporáneas, pues la demanda expresada por los ciudadanos de un fortalecimiento de la democracia directa se manifiesta con fuerza en las aglomeraciones urbanas, donde las reformas institucionales pueden experimentarse con bastante facilidad. En la mayoría de los países considerados en la obra D émocraties urbaines, la democracia local es hoy de naturaleza esencialmente representativa. En la hipótesis de una institucionalización reforzada de la participación directa de los ciudadanos, podemos preguntarnos cómo evolucionará el papel de los electos en las asambleas municipales: ¿serán las víctimas de innovaciones institucional es que consagran la acentuación de la influencia de los ciudadanos con fines de participación creciente y que consolidan la posición de las burocracias para mejorar la eficiencia de los servicios urbanos?

¿Q ué puede aportar, en definitiva, la comparación al estudio de la democracia urbana? Las preguntas que hemos planteado ya han sido objeto de algunos trabajos a escala nacional sobre los sistemas políticos. Los datos y los resultados empíricos de que disponemos para las ciudades son, por el contrario, muy limitados y, cuando existen, están muy dispersos, son heterogéneos 0 están recogidos a un nivel de agregación demasiado globalizante. A parte de los Estados U nidos y de Gran Bretaña, los países incluidos en esta obra casi nunca han dado lugar a investigaciones completas de la estructura y del funcionamiento de la democracia en sus grandes ciudades. Por tanto, es fundamental tratar de comprender en qué medida los procesos de cambio social, económico y cultural producen efectos similares o no en la democracia urbana en contextos nacionales diferentes y cómo esos efectos pueden variar, más o menos, en el interior de cada uno de los países considerados.

Contrariamente a la mayoría de los estudios publicados anteriormente - ya sean excesivamente generales y descriptivos en su enfoque, ya estén basados en uno o en un número restringido de casos- , las diferentes contribuciones se apoyan en un entramado común de análisis y en la explotación de un material empírico original y sustancial cuya misma constitución ha necesitado «paciencia y bastante tiempo» por parte de los diferentes investigadores asociados. Uno de los principales aportes de los datos utilizados - socio-demográficos, electorales, políticos o de outputs de políticas públicas- reside en su personalización por ciudad, en la presentación de series que abarcan varios decenios y en la construcción de índices que permiten comparaciones intra einternacionales entre el electorado y los sistemas de partidos urbanos. 


\section{La búsqueda de una nueva legitimidad de acción}

Si tomamos en consideración a las regiones urbanas occidentales, los problemas que éstas conocen desde hace veinte años - con graduación diferente pero que afectan no sólo a las regiones de tradición industrial - no cesan de acentuarse y exacerbarse. En efecto, lejos de apaciguarse, los conflictos socio-políticos que en ellas se manifiestan se hallan expuestos a un proceso de diferenciación e intensificación creciente. La tesis de BELL (1960) del fin de las ideologías, al igual que la de KIRCH HEIM ER (1965) de la atenuación de los conflictos en las democracias industrializadas, o la estrechamente relacionada con ésta de una «astenia» de la democracia como resultado de una conflictividad insuficiente, no se han confirmado y parecen estar más bien invalidadas por la realidad. Aparecen nuevas divergencias que se superponen a las más antiguas, dando lugar a situaciones caracterizadas por una estructura de conflictos cada vez más diversificados - con el aumento reciente, como subraya H IRSCHM ANN (1995) de conflictos indivisibles 0 either or conflicts, opuestos a los conflictos divisibles, o more or less conflicts- que dan lugar a compromisos delicados y generalmente inestables.

Es en torno a esta hipótesis central de una tendencia a la acentuación de los conflictos socio-políticos sobre la que es pre ciso analizar las transformaciones de la democracia en las áreas metropolitanas, en el entendimiento de que este aumento de la conflictividad puede a la vez actuar como «aglutinante»y como «disolvente» de la sociedad. No se trata de aprobar un amplio elenco normativo ni de argumentar a favor de la integración supramunicipal 0, a la inversa, de subrayar en la óptica del Public Choice las ventajas unidas al mantenimiento de las pequeñas unidades democráticas. Un enfoque mucho más adecuado consiste en identificar y analizar los conflictos fundamentales que estructuran las posiciones y las estrategias de los actores implicados para resolverlos, así como su sucesión y su combinación en el tiempo.

Se pueden distinguir, pues, tres tipos de conflictos. Los conflictos territoriales surgidos de la dominación históricamente determinada en el seno de una región urbana de la ciudad-centro sobre los municipios periféricos. En muchos casos, la posición inicialmente cuasi monopolística de la antigua ciudad con respecto a su entorno no ha cesado de reducirse en el curso de los últimos decenios, que han estado marcados en países como los Estados Unidos por un éxodo masivo de los ciudadanos y de las empresas hacia la periferia. Pero los conflictos actuales sólo pue den interpretarse a la luz del desarrollo específico durante un largo periodo de una trama compleja de cambios, de percepciones y de representaciones recíprocas entre el centro y la periferia. En determinadas regiones urbanas europeas, principal mente en Alemania y en Italia, la preeminencia de la gran ciudad se encuentra atemperada en gran parte por una larga tradición de autonomía y de influencia de las ciudades antiguas que la rodean. Aunque sea la capital y con mucho la ciudad más grande del Land de Bade-Wurtemberg, Sttuttgart continúa siendo considerada con un cierto desprecio como una «ciudad advenediza» que, contrariamente a sus vecinas más próximas Ludwigsburg o Esslingen, no se distinguía hasta el siglo pasado ni por la amplitud de sus recursos ni por cualquier otra proyección.

Los conflictos territoriales se han acrecentado y diferenciado en general de manera considerable en el curso de los últimos años. Una competencia económica y política, a menudo feroz, enfrenta ya a unas ciudades cada vez menos «centros», a municipios que se niegan a ser sólo suburbios o ciudades satélites. N unca la tesis de la explotación fiscal ha sido tan sistemática y ritualmente esgrimida en un sentido o en otro, en ataque 0 en defensa de las grandes ciudades, a medida que se experimentan proyectos de sistemas de distribución equitativa de recursos intercolectivos, como en Francia, o redefinidos sobre la base de una larga práctica, como en Alemania. A estos conflictos de redistribución de recursos económicos y fiscales se unen tensiones socio-territoriales engendradas por una acentuación, principalmente en Europa, de los procesos de segregación social, étnica, de edad o religiosa. Así pues, asistimos a un acercamiento de los modelos europeo y americano de evolución de urbanización, caracterizada por una polarización socio-espacial creciente a escala tanto del municipio como de la metrópolis. Cualesquiera que sean las medidas utilizadas (riqueza, estilo de vida), este movimiento de diferenciación constituye una de las características más fuertes de la evolución reciente de las ciudades occidentales (FrIEDRICHS, 1995).

Una segunda categoría de conflictos se sitúa en torno a los retos socio-políticos que transcienden frecuentemente las de pendencias territoriales. También se han hecho más complejos a lo largo de los últimos años, en la medida en que ya no pue den ser reducidos a una sencilla y clara oposición entre los intereses del capital y los del trabajo, aunque los promotores más activos de fórmulas de institucionalización de gobiernos metropolitanos procedan y sean seleccionados de manera privilegiada entre los responsables de las grandes y medianas empre sas, reagrupados o no en el seno de las cámaras de comercio 0 de organizaciones corporativas. La divergencia actual más de terminante no se apoya en un antagonismo entre clases sociales, sino en una divergencia radical de concepciones del desarrollo económico entre los grupos partidarios de un crecimiento regional fuerte y continuo (los líderes del sector económico, principalmente) y los partidarios de los growth limits, es decir, de un dominio controlado de la extensión de las 
actividades económicas e inmobiliarias (representados, en particular, por las asociaciones de defensa del medio ambiente y las asociaciones de barrio). Nuevos retos - entre los que figura igualmente la integración social y política de las minorías étnicas- y los conflictos que engendran, dominan, cada vez más, la agenda de las políticas urbanas.

La tercera línea de discrepancia es de orden ideológico y se basa en la oposición de los programas y estrategias de las organizaciones de los partidos. Su pertinencia varía según los países - es apenas diferenciable en Estados Unidos, lo que explica que, en general, apenas sea mencionada por los politólogos americanos- y los contextos territoriales. No o se confunde necesariamente con las otras dos categorías de conflictos, aunque los tres tipos de discrepancias interactúan. Así pues, de esta manera se ha podido demostrar la oposición total de los intereses y de las estrategias defendidos, por una parte, por los principales representantes de la economía de la región urbana de Stuttgart y, por otra parte, por una fracción influyente de la CDU, el partido mayoritario en el Land a nivel de la aglomeración, representada por la corriente más conservadora (H OFFM AN N MARTIN OT, 1995).

Una vez más, nuestra hipótesis principal es la de una tendencia a la «sofisticación conflictual» que se manifiesta, en primer lugar, por una ampliación de la gama de partidos y de formaciones políticas representados en las asambleas locales, así como de sus programas de acción. Las nuevas organizaciones, principalmente ecologistas y de extrema derecha, compiten, ahora, en la mayoría de las ciudades europeas con los partidos tradicionales de la derecha, del centro y de la izquierda. Estas nuevas organizaciones alteran la tradicional dominación uni 0 bipartidista y contribuyen a fragmentar el sistema local de partidos, de lo que es ejemplo ilustrativo desde los años ochenta la multiplicación de los hung authorities en Gran Bretaña, 0 bien la elección de un número creciente de tenientes de alcalde ecologistas en las ciudades alemanas. No es solamente el sistema de partidos, sino también sus propios componentes los que han sido sometidos a este proceso de diferenciación ideológica y estratégica a través de la formación de facciones o de clanes intrapartidarios que merma incluso la cohesión de formaciones consideradas como las más sólidas y jerárquicamente mejor organizadas.

La estructura de estos tres tipos principales de conflictos no es ni unilateral ni inmutable. En otras palabras, no permite comprender, ni incluso predecir el posicionamiento y el papel de los diferentes actores así como los compromisos institucionales obtenidos, si no se especifica previamente el periodo y el sistema político nacional existentes, así como las políticas públicas tomadas en consideración según persigan prioritariamente unos objetivos de prestación, de redistribución o de de sarrollo económico.

\section{Las mutaciones contemporáneas de las democracias urbanas}

\section{El declive de lo que significa el momento electoral}

Las formas tradicionales de compromiso político tienden a declinar. Bajo el efecto de una territorialización creciente de los retos, los procesos de designación de los representantes políticos urbanos escapan cada vez más a unos determinantes de origen nacional, de tal manera que la interpretación en términos de nacionalización de las elecciones se revela hoy en día mucho menos pertinente. A pesar de ese significado relativamente más importante de la política territorial, el abstencionismo electoral se incrementa sobre todo desde los años ochenta en la mayoría de los páses europeos, hayan conocido o no reformas descentralizadoras, siendo uno de los efectos supuestos, sin embargo, el refuerzo del sentimiento de los ciudadanos de pertenencia a sus comunidades locales. Sin embargo, esta tendencia a la baja concurrencia a las urnas está lejos de ser uniforme, ya que sigue habiendo fuertes fluctuaciones inter e intranacionales. Cada vez es más baja la participación en la elección de los concejales locales en Estados U nidos (sólo un cuarto de los electores potenciales en las ciudades), en Suiza y en Gran Bretaña, mientras que la concurrencia a las urnas sigue siendo más elevada en Escandinavia, en Italia 0 en Francia. A partir de los datos utilizados que se re fieren al conjunto de los municipios de la Francia metropolitana de más de 20.000 habitantes y de los distritos ingleses de más de 25.000 habitantes, hemos podido identificar varios factores que influyen de manera independiente y determinante sobre el nivel de participación en las elecciones municipales: en estos dos páses el nivel de participación es tanto más elevado cuando el municipio se caracteriza por una estabilidad espacial de su población, una proporción importante de comerciantes, industriales y propietarios y una competencia política intensa (H ofFM An NM ARTINOT, 1996). El civismo electoral varía igualmente en función de las culturas políticas regionales o locales dominantes. En las regiones mediterráneas de Francia, la estructura clientelar de los grupos de notables sostiene una movilización más elevada para los escrutinios locales que para los nacionales, exactamente como ocurre en Japón. Incluso, hoy en día, estas variaciones re gionales de configuración de val ores y de tradiciones políticas no son tomadas suficientemente en cuenta en la mayoría de los análisis electorales, en la medida que éstos preconizan una homoge neización de los modos de socialización y de los marcos de experiencia de los individuos y se basan en muestras llamadas representativas de una población descontextualizada. El enfoque 
en términos de culturas políticas territoriales debería permitir profundizar en la comprehensión de las fluctuaciones de la participación electoral, pero ello implica el recurso preferente a análisis contextuales, combinando la explotación de datos asociadose individuales, cuyos costes de producción son, desgraciadamente, muy disuasorios.

Una transformación destacada de estos últimos años es la creciente diferenciación del registro de los significados de la no participación electoral. Tomando la tipología de DALTON de cuatro grupos principales de electores definidos en función de su interés por la política (I) y de su proximidad en relación a los partidos (P), aparece que dos de estas categorías alimentan cada vez más el fenómeno del abstencionismo, en particular en las grandes ciudades: los apolíticos (I - / P - ) y los partidarios (I + / P - 2). Los apolíticos representan esta parte de la población que no confía en la capacidad de los mecanismos democráticos para mejorar el nivel de vida individual y las condiciones del bienestar social. No participan generalmente o lo hacen de manera excepcional en los escrutinios electorales. Su proporción no ha cesado de crecer en los países occidentales a medida que las olas de reestructuración económica han condenado a un número cada vez más importante de individuos al paro, a la precariedad, a la marginalidad o a la exclusión social. Para estos perdedores de la modernización, el voto está prácticamente privado de su funcionalidad a la vez simbólica (manifestar su adhesión a una comunidad social y de valores) e instrumental (expresar sus preferencias a fin de influir en el curso de las políticas públicas). El aumento progresivo del segundo grupo de ciudadanos invalida de alguna manera el modelo del SES (Socio-Economic Standard), según el cual el nivel de instrucción y de riqueza constituye el vector esencial de una participación política más activa. No solamente los resultados de diversas investigaciones Ilevadas a escala territorial contradicen esta relación, sino igualmente la evolución general del electorado de las democracias occidentales desde los años 80 revela un aumento del abstencionismo a pesar del incremento del nivel medio de instrucción. La interpretación fundada sobre el SES sólo es parcialmente válida para explicar la participación electoral. Ignora, en efecto, el proceso de «sofisticación» de las prácticas y estrategias políticas de esta clase de electores instruidos, más instrumentales en sus elecciones que fieles o leales respecto a una formación partidista determinada y más exigentes en lo que se refiere a las realizaciones de sus representantes. Estos ciudadanos más estrategas y racionales en la expresión y la defensa de sus preferencias se dirigen, por consiguiente, a las urnas menos por sentimiento de obligación cívica que por voluntad de seleccionar el programa político que mejor corresponde a sus necesidades e intereses. El elector consumidor manifiesta así un comportamiento variable y diferenciado, condicionado en gran manera por la estructura de la oferta electoral: según la gama de «productos» que se le ofrezcan decidirá hacer su elección o reti- rarse (abstencionismo intermitente) y dará más significado al contenido de los programas 0 al atractivo de los candidatos que a los slogans partidarios (split-ticket voting). De hecho, todo parece ocurrir como si los «electores sofisticados», que habitan principalmente en las grandes aglomeraciones urbanas, recurriesen a toda una gama de modos de expresión de sus preferencias, entre las cuales el voto sería trivial, mientras que entre el resto de la población conservaría una dimensión superior y privilegiada. Comentando una encuesta de opinión llevada a cabo sobre las percepciones del gobierno local noruego, H AN SEN confirma esta evolución: «existe un efecto de sustitución entre el voto en las elecciones y otras formas más directas de participación política, de tal manera que cuanto más importante es el municipio, menos ciudadanos votarán y participarán más en otras arenas políticas» (H ANSEN, 1994).

\section{La recomposición de los partidos y de los sistemas de partidos}

El declive de las diferencias de clase y de religión ha ocasionado en numerosos países una transformación profunda de los partidos tradicionales, de sus efectivos, de su electorado (cada vez menos (eal), de sus organizaciones y de sus estrategias. LoS efectivos han ido a menos en los Países Bajos - el 10\% de la población adulta en 1960, el 2-3\% hoy- y tienden a bajar en otros países.

Para los partidos y los líderes políticos, el comportamiento de sus electores está llegando a ser mucho más difícil de prever y de interpretar. De ello dan testimonio los valores de la estructura y de la evolución de tres índices que se utilizan clásicamente en sociología electoral comparada: Ios de volatilidad electoral (índice de PEDERSEN), de fragmentación (índice de RAE eíndice de LAAKSO y TAAGEPERA) y de capacidad integradora del sistema de partidos urbanos. En términos generales, se observa en la gran mayoría de los sistemas políticos urbanos una volatilidad electoral superior, una creciente fragmentación (aumento del número de partidos efectivos) y una menor capacidad integradora de los partidos tradicionales.

Así, en la Francia de los años 1971-1995, los sistemas de partidos urbanos reflejan la lógica bipolar izquierda/derecha del enfrentamiento político, favorecido por unas reglas electorales con predominio de la mayoría. La evolución del número de partidos efectivos cal culada a partir del índice de LAAKSO y TAAGEPERA (1979) indica que esta medida está cada vez más cerca de 3, e incluso más próxima a 202,5 , es decir, de una estructuración bipartidista de las fuerzas políticas, en varias ciudades como Angers, Limoges, N antes o Reims. Sin embargo, a partir del final de los años ochenta, asistimos a un movimiento de fragmentación del sistema de partidos, habiendo aumentado el índice en una media regular entre 1983 (2,53), 1989 (3,12) y 1995 
$(3,56)$. M ientras los sistemas de partido muy dominantes, caracterizados por un número de partidos efectivos inferior a 2, existían en 1971 (Le H avre, Perpiñán, Reims, Tours y Villeurbanne), en 1977 (Le Havre y Nimes) y en 1983 (Burdeos, Boulogne-Billancourt, Dijon, M etz y Tours), desaparecen a partir de 1989. En las elecciones municipales siguientes de 1995, el índice sobrepasa el valor de 4 en diez grandes ciudades, eincluso de 5 en cuatro de ellas, lo que era excepcional hasta finales de los años ochenta.

D esde un punto de vista metodológico y de estrategia de investigación comparada, la presentación, la utilización y la comparación de esos tres índices se revelan particularmente esclarecedores. El uso de este tipo de instrumento permite superar los enfoques ya sean demasiado generales - por no estar basadas sobre datos empíricos precisos- ya sean demasiado particulares por basarse en estudios de casos, municipales y/o nacionales.

\section{El progresivo reconocimiento del papel activo del ciudadano en la política urbana}

El crecimiento del papel y de las opciones de los individuos constituye un leitmotiv de los nuevos conceptos del gobierno urbano. Tradicionalmente relegado al puesto de elector que no interviene en los procesos políticos nada más que para designar sus representantes a intervalos relativamente largos - generalmente entre cuatro y seis años- la implicación del ciudadano está hoy muy solicitada. El dilema de la asociación de los ciudadanos condiciona, sin embargo, la transformación de la democracia urbana contemporánea: los sistemas territoriales existentes y sus dispositivos institucionales no les reconocen más que un lugar muy limitado, mientras que los mecanismos aptos para favorecer una transmisión más completa de sus preferencias no garantizan necesariamente la mayor participación posible.

En el curso de los últimos años, la diversificación y la acentuación de los conflictos urbanos han ocasionado una extensión de la politización de los retos durante mucho tiempo considerados como esencialmente técnicos o neutros. Lejos de decaer, el interés por la política local de los ciudadanos se ha reforzado en los países industrializados. En varios países, como N oruega 0 los Estados U nidos, encuestas de opinión rigurosas - como las del organismo americano N ORC (N ational O pinion Research Center) - demuestran desde los años 1960 a 2000 un compromiso más fuerte por parte de los ciudadanos en los asuntos locales (participación en asociaciones, contactos con los elegidos y los funcionarios... ) (cfr. VERBA y otros, 1995). En Europa, el creador de incertidumbre más temido ahora por los responsables urbanos ya no es tanto el Estado y sus representantes, los banqueros o la oposición política institucional, como los diferentes movimientos, grupos y asociaciones surgidos de la pobla- ción. Entre los testimonios abundantes en este sentido, citamos un fragmento de una reflexión del alcalde de la ciudad alemana de Friburgo - cuyo perfil es predominantemente post-materialista- sobre la generalización de la cultura llamada de N IM BY smo (N ot in M y Backyard 3 ): «... El consejo municipal elegido no haría jamás nada como es debido. Vemos aquí cómo el principio representativo se encuentra conscientemente (o inconscientemente) vaciado de su contenido, siendo el objetivo establecer un intercambio con los de «abajo». ¿Pero quienes son estos portavoces de la base? ¿Valen más que los consejeros municipales, los elegidos por el conjunto de la ciudad?» (Bö H M, 1993: 106).

Se está fomentando o experimentando una nueva ciudadanía, que modifica el contenido y el reparto tradicional de los papeles ejercidos, los de elector, de contribuyente y de destinatario de servicios. Formas más directas de participación y de comunicación, que diversifican notablemente los modos de interacción entre electores y lideres, están sustituyendo, cada vez más, al voto. Los destinatarios de los servicios se hallan cada vez más asociados a la evolución de las modalidades de su prestación y son considerados más como clientes activos que como usuarios cautivos. Esta transformación en curso favorece, por tanto, la he terogeneización y la desmultiplicación de los papeles del ciudadano, que cada vez más es tratado como un consumidor ilustrado (idealmente) de servicios urbanos.

Aunque a veces sea bastante poco visible, este proceso de implicación creciente de los ciudadanos en las decisiones, impulsado por los municipios, aparece más profundo que el movimiento de naturaleza ideológica, de límites difusos y mal controlados, de los años 70. Constituye un reto de legitimidad fundamental para los responsables municipales que ya no pueden contar con la lealtad clásica de su electorado. D e ahí el desarrollo de la coproducción de servicios y el refuerzo de la consulta a los ciudadanos y a las asociaciones en las políticas de urbanismo, en las políticas escolares o bien en las de tercera edad. Esta evolución no podría esconder, sin embargo, la imposición de un filtro social muy frecuente. La sobrerrepresentación de la clase superior en numerosas asociaciones municipales ilustra la fórmula imaginativa del politólogo americano Eric E. SCH ATTSCH nEIDER: «EI fallo del paraíso pluralista es que el coro celestial canta con un tono muy chic».

Una autonomía local desarrollada supone la existencia de un gobierno local eficiente, que disponga de recursos suficientes para asegurar lo que puede realizar mejor que cualquier otro aparato institucional: una prestación de servicios que las comunidades locales están teóricamente en condiciones de asegurar óptimamente, por su proximidad a la población, su capacidad de coordinar las acciones públicas y de integrar los diferentes re tos y sus procedimientos de tratamiento. H oy como en el pasado, algunos debates han llevado a oponer, a veces de manera sis- 
temática, la búsqueda de la eficiencia y la valorización de la de mocracia, como si esos dos val ores fueran excluyentes el uno del otro. En el fondo y en numerosos contextos, aparecen más bien como complementarios, lo que parecen haber comprendido el gobierno central británico, al igual que numerosos alcaldes actuales de grandes ciudades europeas que desean favorecer la re conciliación de sus ciudadanos con la política urbana asociándoles estrechamente con la mejora de la calidad de los servicios (CLARK y H OFFMANn -M ART INOT, 1998). La evolución es desde ese punto de vista sorprendente en los Estados Unidos: la separación clásica desde el comienzo del siglo xx entre los demócratas tradicionales, por un lado, defensores de la política con sus enfrentamientos de clases y grupos étnicos, sus luchas de partidos y de maquinarias, sus conflictos de programas y de ideologías, y los reformistas, por otro, en pugna por una gestión pública despolitizada y desideologizada, sobria, racional y eficiente, parece haberse atenuado considerablemente y haber sido remplazada por un sincretismo de gestión y de populismo. Los nuevos líderes negros, como los al caldes de Cleveland o de Baltimore, M ichael White y Kurt Schmoke, están lejos de la línea de activismo militante de los «paladines de la causa racial» de los años sesenta, como Colman Young en D etroit o M arion Barry en Washington, y aparecen más como «tecnopolíticos» (EISIN GER, 1998).

\section{La diferenciación de escalas de la democracia urbana}

Nos encontramos aquí con una cuestión fundamental: la de la relación entre el tamaño de una comunidad y democracia. ¿Cómo puede organizarse y funcionar una democracia viva y suficientemente descentralizada en una gran ciudad? D ebemos conceder una especial importancia a esta cuestión cuya evocación comienza con el Gran Berlín de los años veinte y sus primeros consejos de distrito, para volver a reflotar en los años 196070 (Bolonia, 0 slo) y resurgir después de manera recurrente, principalmente en España (Rodriguez Álvarez, 1996). ¿D ónde estamos hoy?

Incluso aunque la timidez reformadora en este campo siga siendo la característica de algunos páises como Francia, al contrario de lo que sucede en otros como los países nórdicos, un cambio en profundidad afecta a numerosas ciudades, incluidas las que arrastran desde lustros una reputación de oligarquía centralizada.

Los estudios más avanzados han sido llevados a cabo en los países nórdicos y el balance que se ha podido sacar de la descentralización inframunicipal, uno de cuyos objetivos principales es reforzar la democracia local estimulando el interés de los ciudadanos, la identificación local y la participación, es moderado. Se gún el politólogo sueco Stig M o NTIN, los ciudadanos, por lo ge- neral, ignoran la existencia de los comités de barrio y no se pue de afirmar que la descentralización inframunicipal haya conducido a mayor participación de los ciudadanos; demuestra, además, que la búsqueda de la eficiencia ha sido sustituida cada vez más como objetivo esencial de esta reforma por un fortalecimiento de la democracia. El equipo de investigadores en ciencias sociales que ha examinado la reforma de G öteborg ha llegado a las mismas conclusiones. La ausencia de efectos sobre la participación democrática ha sido en cambio contestada por el equipo de politólogos de Estocolmo dirigido por Premfors, al término de su evaluación de la experiencia de tres comités de barrio de Estocolmo entre 1990 y 1995. En lo que se refiere al «modelo democrático fuerte», estiman que los efectos de esta reforma se han revelado positivos.

Aunque los efectos de la descentralización inframunicipal sobre la participación de los ciudadanos pueden parecer pobres, numerosas evaluaciones indican en cambio que esta reforma ha contribuido a modificar el papel de sus representantes de barrio. Éstos se identifican y son aún más identificados en sus barrios. $H$ an adquirido un mejor conocimiento de los contextos locales y mantienen relaciones más fructíferas con los ciudadanos. Ade más, cuando se les compara con los miembros de los antiguos comités sectoriales, los concejales de barrio tienen un enfoque más «generalista» de la política y son más independientes de la burocracia especializada.

Incluso en un país como Gran Bretaña, donde la asociación directa de los ciudadanos es más difícilmente reconocida, la extensión de la participación, en términos de amplitud de la arena donde la población puede expresarse y donde los responsables deben escuchar o explicarse, tiende a progresar a pesar de todo. Los consejos de comunidad, tales como los de Birmingham, se hallan más extendidos que los comités de barrio del tipo de Tower $\mathrm{H}$ amlets en Londres, pero todas esas estructuras tienden al mismo objetivo: escuchar a los habitantes y reducir la distancia entre ellos y sus representantes.

\section{La democracia en peligro de fragmentación o cómo resolver desde arriba}

En el conjunto de los países considerados, la democracia urbana pasa por un proceso de fragmentación creciente que se manifiesta tanto territorialmente - a la ciudad la sustituye la me trópoli balcanizada, por una parte, e incluso los barrios/distritos, por otra- como funcionalmente - por la burocratización, especialización funcional y desarr ollo de aislamientos sectoriales- como políticamente. De ahí la constatación cada vez más amarga de la ausencia de un piloto en el avión, de un líder del grupo municipal capaz de integrar, reagrupar, sintetizar, decidir y representar. 
El reto es enorme en las grandes ciudades, donde se trata no sólo de reforzar el poder de integración del alcalde, sino también de acrecentar su legitimidad. El perfil del alcalde francés tradicional, dotado de una influencia a menudo desmesurada, sirve a estos efectos de referencia positiva y negativa a la vez. Las diferentes reformas en curso o ya puestas en práctica en Europa se orientan a dotar al alcalde, por una parte, de un peso y de una representatividad política suficientes para permitirle negociar con éxito a nivel de Estado e incluso, más generalmente, a escala extramunicipal, a fin de aparecer como un líder representativo; y, por otra parte, de una fuerte legitimidad, manifestación de movilización y de participación activa de la población, asegurándole una posición de líder político; y finalmente de un estatuto de profesional eficaz de la administración y de la gestión pública, capaz de dirigir el grupo complejo y polimorfo de la burocracia municipal como líder de la organización.

Este reto es 0 ha sido central en el curso de los últimos años, tanto en Alemania como en Italia, en los Países Bajos, en los países nórdicos o en Gran Bretaña. La solución recomendada y progresivamente adoptada ha sido el establecimiento de un régimen de tipo semipresidencial por instauración de la elección directa del alcalde, en definitiva, una solución desde arriba. Allí donde se ha puesto en marcha una reforma semejante, como en Italia 0 en Alemania del Norte 0 del Este, los efectos democráticos en términos de ampliación de la influencia de los ciudadanos, de responsabilidad política y de accountability son patentes. Pero una innovación tal no ha sido realmente introducida más que en Italia, en un contexto de crisis profunda del sistema político; no ha sido sino generalizada a partir de los Länder del sur en Alemania, experimentada con prudencia en Gran Bretaña y ocultada u olvidada en los otros países. Esta cuestión de la construcción de un líder elegido directamente por la población se plantea igualmente de manera crucial a escala de las metrópolis.

Los retos asociados a la fragmentación territorial han tenido tendencia a exacerbarse en los últimos años en la mayoría de las regiones urbanas occidentales, ocasionando la metropolización de un gran número de problemas municipales y la reactualización de las perspectivas de reforma institucional casi abandonadas desde finales de los años setenta. Sin embargo, la natural eza de los retos ha evolucionado sensiblemente. La búsqueda del óptimo dimensional para la gestión de los servicios públicos locales en el marco de una modernización integradora de las acciones del Estado y de las entidades territoriales es la preocupación central. En cambio, las fórmulas de cooperación más o menos integradas establecidas en los años 1960-70 demuestran manifiestamente sus insuficiencias en la regulación de retos cada vez más conflictivos, tales como la planificación urbana, el desarrollo económico, la acentuación de las dispari- dades socioeconómicas, la gestión de los grandes equipamientos colectivos o la protección del medio ambiente. Las comunidades urbanas francesas, como las Regi onalverbände alemanas, se resienten de ejercer unas competencias sobre unos territorios hoy en día superados por la extensión de las regiones urbanas, y quizá sobre todo de no estar dotadas de una legitimidad supramunicipal que les permita superar los conflictos y los bloqueos provocados por la afirmación de los diferentes intereses municipales representados.

Contrariamente a un cierto número de estereotipos todavía vigentes y ampliamente compartidos con sus vecinos, incluso Suiza - y más particularmente al gunos de sus cantones- considerada visceralmente ligada a la autonomía de las pequeñas comunidades y a la conciliación entre democracia y eficiencia, se halla comprometida desde finales de los años 1990 en una importante reestructuración de su mapa territorial, apenas modificado desde hace 150 años. M uchos observadores exteriores se habían quedado cómodamente con esta imagen de Epinal, me jorada por los partidarios de la Public Choice, según la cual los pequeños municipios suizos funcionaban óptimamente a un coste muy competitivo respecto a las comunidades de otros países europeos. Esta representación bucólica y sosegada de una Suiza globalmente rica y protegida de las grandes corrientes de cambio social - aparte sin duda de al gunas grandes ciudades «con problemas» o de ciertas regiones rurales desfavorecidasapenas se corresponde con la realidad. Un tercio de los municipios ha presentado un déficit presupuestario en el curso de los tres últimos años (H OFFM AN N-M ARTIN OT, 2001).

¿Por qué Suiza sale hoy de su inmovilismo en el campo de la organización municipal? Y ello cuando incluso ciertos procedimientos, la fusión en particular, han sido además pospuestos, incluso oficial mente abandonados. Suiza está quizá adelantada a su tiempo. Parece, de hecho, compartir tendencias comunes a los otros países industrializados: el aumento de las funciones que los municipios deben asegurar por la presión insistente de las autoridades estatales (movimiento interpretado como una recentralización, ver KJELLBERG, 1995) y, al mismo tiempo, una expansión y una flexibilización de sus capacidades de acción, que van en el sentido de la descentralización. Esta dialéctica de asociación más o menos equilibrada con un Estado menos visible pero no menos dirigente nos obliga a revisar profundamente la imagen de una Suiza tierra de plenitud de la autonomía local: ¿quién sospecharía que el 38\% de sus cantones pueden autoritariamente fusionar municipios? Es probable que numerosas autoridades cantonales tengan interés en ver emerger unas unidades locales aptas para negociar sus asuntos sin mendigar sistemáticamente un apoyo, para llegar a una progresiva desimbricación de los niveles de acción (Entflechtung). Pero las estrategias de los actores cantonales conocen quizá un grado elevado de diferenciación. 
* Profesor del Instituto de Estudios Políticos de la Universidad de Burdeos y uno de los fundadores de la red EURO LOC, y, también, D irector de Investigación del Centre Nacional de Recherches Scientifiques.

Traducción española de la versión original francesa de Elisa Rom ero SEBASTIÁn. $1 \mathrm{H}$ enry Bäck, Folke Johansson et H elge Larsen (Países N órdicos), Bas D enters (Países Bajos), 0 scar W. G abriel (Alemania), Vicent H offmann-M artinot (Francia), N obufumi Kawasaki (Japón), Annick M agnier (Italia), Louis Q uesnel (Canadá), H ank Savitch (Estados Unidos), Elinor Scarbrough (Reino Unido).
$2 \mathrm{Q}$ ue D ALTON distingue unos partidarios protocolares/rituales (I - / Pt) y unos partidarios cognoscitivos $(I+/ P+)$.

3 La expresión inglesa N IM BY (not in my back yard: «no en mi patio trasero») 0 N IM BYsm (»nymbismo») hace referencia a la política insolidaria de numerosos municipios, consistente en no aceptar usos del territorio no deseados por sus gobernantes o su población, tales como polígonos de viviendas sociales, desviando su implantación hacia municipios más alejados.

\section{Bibliografia}

Association des M aires de Grandes Villes de France (1994). M on pays, cest la ville. Le manifeste des M aires de G randesVilles de France, París, Bernard Grasset.

BeLL, D aniel. (1960). The End of I deology. N ew York: Free Press.

BöH ME, Rolf. (1993). Je mehr wir haben, desto mehr haben wir zu wenig. Bemerkungen aus dem politischen Alltag. Bonn: D ietz.

Canales Aliende, José M anuel. (2000). «Los retos de la nueva Gestión Pública Local en España», Revista de Estudios Locales, 39, junio: 34-41.

Clark Terry, N. y H offm ann-M artin ot, Vincent. (Eds.). (1998). The N ew Political Culture, Boulder, Co., Westview Press,

Dalton Russell, J. (1984). «C ognitive M obilization and Partisan D ealignment in Advanced Industrial D emocracies», Journal of Politics, 1984, 46: 264-284.

EISINGER, Peter. (1998). «City Politics in an Era of Federal D evolution», U rban Affairs Review, Vol. 33, número 3, January, pp. 308-325.

FriedRICHS, Jürgen. (1995). Stadtsoziologie. O pladen: LesketBudrich.

Gabriel, Oscar W., H OfFmann-M artin OT, Vincent y Savitch, Hank (Eds.). (2000). Urban D emocracy. O pladen: Leske+Budrich (Städte und Regionen in Euro$\mathrm{pa}, 1)$.

H AN SEN, Tore. (1994). «Local Elections and Local Government Performance», Scandinavian Political Studies, Vol. 17, número 1: 1-30: 24.

Hirschman Albert, 0. (1995). A Propensity to Self-Subversion. Cambridge, M ass:: $H$ arvard University Press: chap. 20

H OFFM AN N -M ARTINOT, Vincent. (2001). «Comparer et mesurer la réforme municipale en Suisse et en Europe», en: Jean-Philippe LERESCHE (bajo la dirección de), Gouvernance locale, coopération et légitimité Le cas sui sse dans une perspective comparée. París: Pédone: 145-163.
H offm an n-M artin Ot, Vincent; Rallings, Colin y Thrasher, M ichael (1996). «Comparing local electoral turnout in Great-Britain and France: M ore similarities than differences?», European Journal of Political Research, 30, September 1996: 241-257.

H OFFM AN N -M ARTIN OT, Vincent. (1995). «II rilancio del governo metropolitano: il caso di Stoccarda», II N uovo Governo Locale, XIII, número 1: 15-43.

KIRCH HeIM ER, Otto. (1965). «D er Wandel des westeuropäischen Parteisystems», PolitischeVierteljahresschrift, 6, Heft 1: 20-41.

KJELLBERG, F. (1995). «The Changing Values of Local Government», The Annals of the American Academy of Political and Social Sciences, número 540, July, pp. 40-50.

Kron AWITter, Georg (H rsg.) (1994). Rettet unsere Städte jetzt! D as M anifest der O berbürgermeister, Düsseldorf, ECON Verlag.

LaAkso, M arkku y Taagepera, Rein. (1979). «The «Effective» N umber of Parties. A Measure with Applications to Western Europe», ComparativePolitical Studies, 1979, Vol. 12, p. 3-27.

Rodríguez Álvarez, JoséM anuel. (1996). «Las juntas de distrito en las grandes ciudades españolas. Un estudio comparado de su organización y de su regulación positiva», Actualidad Administrativa, XXXI, 27, 1-7 julio: 525-588.

Tem PLE, M ichael. (1991). «Changing Political Environment and Bureaucratic Practice: A Case Study of British Local Government», en: RICHARD, Balme y H OFFMANN-M ARTINOT, Vincent (Eds.), Local and Regional Bureaucracies in Western Europe, Talence: Les Cahiers du Centre d'Etude et de Recherche sur la Vie Locale, número 2, 295, pp. 217-244.

Sydney, Verba; Lehman Schlozman, Kay y Brady, Henry E. (1995). Voice and Equality. Civic Voluntarism in American Politics. Cambridge, M ass.: H arvard University Press: 49-96. 